\title{
Simple model for the rf field amplitude dependence of the trapped flux sensitivity in superconducting rf cavities
}

\author{
Sergio Calatroni* \\ CERN, 1211 Geneva 23, Switzerland \\ Ruggero Vaglio \\ Dipartimento di Fisica, Università di Napoli Federico II, CNR-SPIN e INFN, Napoli, Italy
}

(Received 2 October 2018; published 4 February 2019)

\begin{abstract}
The improvement of the performance of superconducting rf cavities has recently motivated a considerable research effort in order to elucidate the effect of trapped magnetic flux on the surface resistance $R_{s}$. In this paper, we show that, by introducing a nonlinear pinning force in the GittlemanRosenblum equations for the rf power dissipation due to a trapped magnetic flux in a superconductor, we can empirically describe the linear dependence on the rf field amplitude $B_{r f 0}$ of the additional surface resistance $R_{f l}$. We also show that the proportionality between the rf-field-dependent and -independent terms $R_{f l}^{1}$ and $R_{f l}^{0}$ and the frequency dependence of $R_{f l}^{1}$ follow naturally from this approach.
\end{abstract}

DOI: 10.1103/PhysRevAccelBeams.22.022001

\section{INTRODUCTION}

The quest for optimizing energy consumption in particle accelerators is motivating the research for increasing the quality factor in superconducting radio-frequency (SRF) cavities, as underlined in recent projects [1,2]. This in turn has motivated a renewed interest in several laboratories to study the effect of a trapped magnetic flux on SRF cavity performance and on the possibilities of minimizing its consequences. The effect of a trapped magnetic flux on the quality factor $Q=\Gamma / R_{s}$, where $R_{s}$ is the surface resistance and $\Gamma$ depends only on the cavity geometry, has been experimentally identified and studied since the earliest developments of SRF cavities [3-5]. The experimental data are generally well described, in particular, at a low rf field amplitude, by an extra additive term $R_{f l}$ to the cavity surface resistance $R_{s}$, having the bilinear form

$$
R_{f l}\left(B_{r f 0}, B_{0}\right)=\left(R_{f l}^{0}+R_{f l}^{1} B_{r f 0}\right) B_{0},
$$

where $B_{r f 0}$ is the rf field amplitude, $B_{0}$ is the trapped external field, $R_{f l}^{0}$ is the zero rf field trapped flux sensitivity, and $R_{f l}^{1}$ is the rf slope of the sensitivity. The first term is typically dominating in the case of bulk niobium cavities, although the second term can become of a comparable order of magnitude at high rf fields, in particular, for

\footnotetext{
*sergio.calatroni@cern.ch
}

Published by the American Physical Society under the terms of the Creative Commons Attribution 4.0 International license. Further distribution of this work must maintain attribution to the author(s) and the published article's title, journal citation, and DOI. high-frequency cavities [6,7]. Interestingly, it was found that cavities made with the sputter thin film technology of niobium on a copper substrate $(\mathrm{Nb} / \mathrm{Cu})$ were much less sensitive to the ambient magnetic field, usually because of a much smaller $R_{f l}^{0}$ compared to bulk $\mathrm{Nb}$ cavities $[5,6]$. Earth's magnetic field is large enough to induce a sensible degradation of the surface resistance, and this motivated the use of efficient magnetic shielding of bulk Nb SRF cavities; conversely, $\mathrm{Nb} / \mathrm{Cu}$ cavities need not be shielded in typical accelerator applications [8]. In recent years, it has also been realized that the expulsion or trapping of magnetic flux depends in large part on the cooldown dynamics and, in particular, on the temperature gradient along the cavity upon crossing the critical temperature $[9,10]$. Although adequate cooldown conditions allow obtaining very large quality factors [11], understanding of the phenomenon has motivated several studies, in particular, as a function of the niobium structure, treatment, and mean free path $[12,13]$. Recent investigations have also focused on the frequency dependence of the effect of trapped magnetic flux [7], confirming and integrating older results [3].

In this paper, we will present a simple model based on a nonlinear extension of the classical Gittleman and Rosenblum (GR) model [14], originally introduced to describe dissipations in thin films due to the motion of a rigid flux lattice under the effect of an rf current as in a forced linear oscillator. Our model reproduces the linear dependence of $R_{f l}$ on $B_{r f 0}$ described by Eq. (1) and the experimental correlation found between $R_{f l}^{0}$ and $R_{f l}^{1}$. We will also show that the available experimental data on the frequency dependence of $R_{f l}^{1}$ can be well described by our model. 


\section{THE GITTLEMAN AND ROSENBLUM MODEL}

The original GR model considers a thin slab with uniform rf currents in its thickness $d$ and small displacements of a rigid vortex lattice due to the small applied rf current compared to the critical current. This last point corresponds to the assumption of a harmonic potential $U(x)=\frac{1}{2} k x^{2}$, where $x$ is the displacement from the equilibrium position and $k$ the elastic constant per unit fluxon length. This results in an equation of motion for a typical flux tube:

$$
\eta \dot{x}(t)+k x(t)=\phi_{o} J_{r f 0} \sin \omega t .
$$

Here $\eta=\phi_{o} B_{c 2} / \rho_{n}$ is the flow viscosity per unit length with $\phi_{0}$ the flux quantum, $B_{c 2}$ the superconductor upper critical field and $\rho_{n}$ the normal-state electrical resistivity, $J_{r f 0}$ is the maximum amplitude of the rf current of angular frequency $\omega$, and $\phi_{o} J_{r f o}$ is the maximum force per unit length exerted by the rf field on the flux lines. The effective mass per unit length $m$ of the fluxons is small, and the acceleration term $m \ddot{x}(t)$ can typically be neglected $[14,15]$, which simplifies the calculations. The flux lattice displacement is then

$$
x_{o}(t)=x_{o} \sin (\omega t-\varphi)
$$

with

$$
x_{0}=\frac{J_{r f 0} \phi_{0}}{\eta \sqrt{\omega^{2}+\omega_{0}^{2}}} ; \quad \operatorname{tg} \varphi=\frac{\omega}{\omega_{0}} ; \quad \omega_{0}=\frac{k}{\eta} .
$$

From the calculated fluxon velocity, it is straightforward to calculate the average power dissipated by the fluxon motion. The surface resistance due to flux motion is then obtained by the standard relation:

$$
P_{s}=\frac{1}{2} R_{f l} H_{r f o}^{2}, \quad \text { with } \quad H_{r f 0}=J_{r f 0} d=\frac{B_{r f 0}}{\mu_{0}} .
$$

The model can also be extended to the case of isolated vortices trapped by pinning centers of radius $r_{0}<\xi$. In this case, the pinning force per unit length is $k \cong J_{c} \phi_{0} / \xi$ with $J_{c}$ the depinning current density [16] and $\xi$ the coherence length. The overall power dissipation is then obtained by summing the individual flux line dissipation. The result is the same as in the original approach:

$$
R_{f l}=R_{n} \frac{B_{o}}{B_{c 2}} \frac{\omega^{2}}{\omega^{2}+\omega_{o}^{2}},
$$

with $B_{0}=n \phi_{0}$ and $R_{n}$ is the normal-state surface resistance $\left(R_{n}=\rho_{n} / d\right.$ for a thin slab of thickness $\left.d\right)$. Note that Eq. (6) in the limit $\omega \gg \omega_{o}$ gives $R_{f l}(0)=R_{n}\left[B_{o} / B_{c 2}\right]$, which reproduces the result of the so-called "static model" [17]. The trapped flux sensitivity $S$ deduced from Eq. (6) is

$$
S=\frac{R_{f l}}{B_{o}}=\frac{R_{n}}{B_{c 2}} \frac{\omega^{2}}{\omega^{2}+\omega_{o}^{2}} .
$$

A crucial parameter of the model is the depinning frequency $\omega_{0}$ that separates the "pinning regime" $\omega \ll \omega_{0}$ from the "flux flow regime" $\omega \gg \omega_{0}$, where the viscous force is larger than the elastic force, resulting in a steep increase of the dissipated power. This gives a possible interpretation on why thin film cavities present a lower sensitivity to trapped flux with respect to the bulk. Indeed, $\mathrm{Nb}$ thin films present a higher depinning frequency in respect to high residual resistivity ratio (RRR) bulk materials so that the condition $\omega \ll \omega_{0}$ is satisfied at typical accelerating cavity frequencies [18].

Though the original GR model was considering very thin films, the model can be extended in principle to film thicknesses smaller than the pinning correlation length $L_{c} \cong 2 \xi \sqrt{J_{d} / J_{c}}$ (here $J_{d}$ is the depairing current density) that can be over $1 \mu \mathrm{m}$ for $\mathrm{Nb}$ films [19].

Various extensions of the model have been recently proposed in the literature [20,21] showing that the GR-type models can fit also the experimental results of pure bulk cavities, though the assumption of perpendicular rigid vortices driven by uniform rf currents seems to be hardly applicable to describe the long flexible vortices trapped in bulk $\mathrm{Nb}$ cavities. Starting from this last consideration, a model of nonrigid fluxons including both bending modes and the nonlocal tension of the vortex line has been recently introduced [22], describing experimental results with very good accuracy $[23,13]$.

It is important to underline here that all the models based on the GR simple picture describe the pinned flux lines under the effect of the rf currents as forced linear oscillators, so that the power dissipation is always proportional to the square of the rf field amplitude; hence, the surface resistance does not depend on $B_{r f}$. In different words, these models can only describe the term $R_{f l}^{0}$ but do not provide a description of $R_{f l}^{1}$. In the following, we will extend the basic GR model, introducing an ad hoc nonquadratic pinning potential (nonlinear pinning force) in order to reproduce the linear $B_{r f}$ field amplitude dependence experimentally observed by many authors [3,5,6,7]. We should mention that a recent different approach also attempts at explaining the dependence on $B_{r f}$, by making use of a mean-field collective pinning potential [24].

\section{NONLINEAR FLUX OSCILLATION MODEL}

Let us assume a nonharmonic pinning potential:

$$
U(x)=\frac{1}{2} k x^{2}-\frac{1}{3} \gamma\left|x^{3}\right| .
$$

This form of the pinning potential is a reasonable approximation of theoretical predictions of the real 

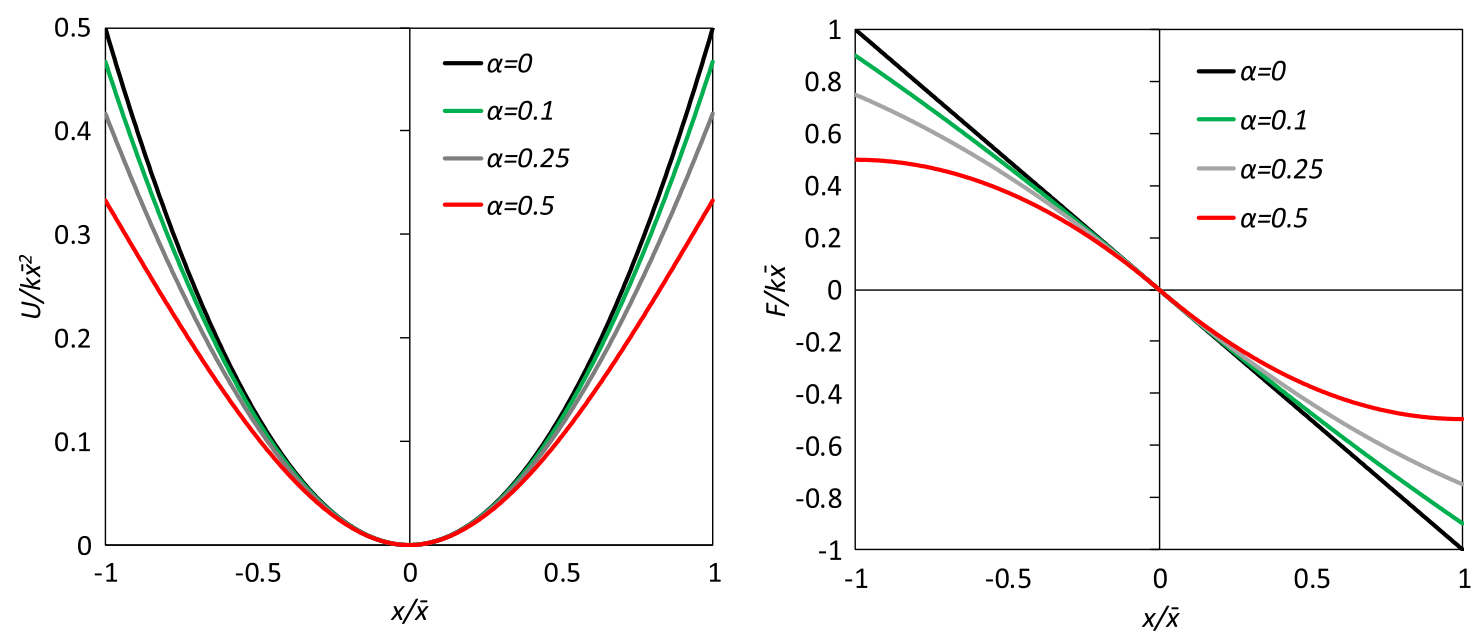

FIG. 1. Plots of $U(x)$ (left) and $f_{p}(x)$ (right) as a function of the relative vortex displacement $x / \bar{x}$ for various values of the parameter $\alpha$.

potential well shape [25]. The pinning force per unit length corresponding to this potential is

$$
f_{p}=-k x+\gamma|x| x .
$$

$U(x)$ and $f_{p}(x)$ are plotted in Fig. 1 for different values of the adimensional parameter $\alpha=\gamma \bar{x} / k$, where $\bar{x}$ is the maximum vortex displacement from the equilibrium position and $\alpha$ represents the ratio of the nonlinear to the linear component of the pinning force at the maximum vortex displacement. It can be seen that, for $\alpha>0.5$, the force is nonmonotonic in $x$, corresponding to a nonphysical behavior.

In the same approximations of the GR model, we need now to solve the nonlinear differential equation

$$
\eta \dot{x}(t)+k x(t)-\gamma|x(t)| x(t)=\phi_{0} J_{r f 0} \sin \omega t .
$$

Equation (10) can be rewritten in terms of the adimensional parameter $\alpha$ :

$$
\frac{1}{\omega_{o}} \dot{x}+x-\alpha\left|\frac{x(t)}{\bar{x}}\right| x(t)=\frac{J_{r f o} \phi_{O}}{k} \sin \omega t .
$$

For a small nonlinear term, i.e., for $\alpha \ll 1$, we can solve this equation by classical perturbation methods, expanding $x(t)$ in powers of $\alpha$. At the first order,

$$
x(t)=x_{o}(t)+\alpha x_{1}(t),
$$

where $x_{o}(t)=x_{o} \sin (\omega t-\varphi)$ is the same solution as for the linear case $(\alpha=0)$, with $x_{o}$ and $\varphi$ already defined by Eq. (4).

By substituting Eq. (12) in Eq. (11) and neglecting the terms above the first order in $\alpha$, we have the linear differential equation for $x_{1}(t)$ :

$$
\frac{1}{\omega_{o}} \dot{x}_{1}(t)+x_{1}(t)=\left|\frac{x_{o}(t)}{x_{o}}\right| x_{o}(t)
$$

where at the same approximation order we can write $\alpha=\gamma x_{o} / k$, since $\alpha=\gamma \bar{x} / k=\gamma\left(x_{o}+\alpha \bar{x}_{1}\right) / k$.

The instant power dissipation due to the single vortex line flow motion is $p_{s f l}(t)=d f_{L}(t) v(t)$, where we have that $f_{L}(t)=\phi_{o} J_{r f 0} \sin \omega t$ is the force per unit length and $v(t)=$ $\dot{x}(t)=\dot{x}_{o}(t)+\alpha \dot{x}_{1}(t)$ the vortex velocity, and to get the power loss we have to multiply for the slab thickness $d$ (as in the GR approach the force is assumed constant along the vortex length). We can now calculate the average power per unit surface $P_{s f l}$ dissipated per cycle by a single fluxon as

$$
\begin{aligned}
P_{s f l}= & P_{s f l o}+P_{s f l 1} \\
= & d \phi_{o} J_{r f o} \frac{1}{T} \int_{0}^{T} \dot{x}_{o}(t) \sin \omega t d t \\
& +d \phi_{o} J_{r f o} \alpha \frac{1}{T} \int_{0}^{T} \dot{x}_{1}(t) \sin \omega t d t .
\end{aligned}
$$

Here, obviously, $P_{\text {sflo }}$ corresponds to the single fluxon power dissipation in the linear GR model.

Expanding $x_{1}(t)$ in Fourier series,

$x_{1}(t)=\sum_{-\infty}^{+\infty} A_{n} e^{i n \omega t} ; \quad A_{n}=\frac{1}{T} \int_{0}^{T} x_{1}(t) e^{-i n \omega t} d t$.

Integration by parts of the second integral in Eq. (14) shows that

$$
P_{s f l 1}=-d \phi_{o} J_{r f o} \alpha \omega \operatorname{Re}\left[A_{1}\right]
$$

Integration of Eq. (13) then gives

$$
\left(1+i \frac{\omega}{\omega_{o}}\right) A_{1}=\frac{1}{T} \int_{0}^{T}\left|\frac{x_{o}(t)}{x_{o}}\right| x_{o}(t) e^{-i \omega t} d t
$$


TABLE I. Coefficients $R_{f l}^{0}$ and $R_{f l}^{1}$ as measured by several authors. The first lines are for bulk Nb, while the bottom lines are for $\mathrm{Nb} / \mathrm{Cu}$.

\begin{tabular}{|c|c|c|c|}
\hline Author & Frequency $[\mathrm{MHz}]$ & $R_{f l}^{0}[\mathrm{n} \Omega / \mathrm{G}]$ & $R_{f l}^{1}[\mathrm{n} \Omega /(\mathrm{G} \cdot \mathrm{mT})]$ \\
\hline Piosczyk $^{\mathrm{a}}[3]$ & $91 / 160 / 290$ & $3.5 / 9.5 / 28$ & $0.35 / 0.55 / 0.9$ \\
\hline Arnolds-Mayer [5] & 500 & 150 & 5 \\
\hline Checchin $^{\mathrm{b}}[7]$ & $650 / 1300 / 2600 / 3900$ & $700 / 1000 / 1500 / 1900$ & $1.6 / 2.6 / 6.1 / 7.4$ \\
\hline Miyazaki [26] & 101 & 3.2 & 0.32 \\
\hline Benvenuti $^{\mathrm{c}}$ [27] & 1500 & $3.3 / 56$ & $0.91 / 4.5$ \\
\hline
\end{tabular}

${ }^{\text {a }}$ Recalculated from data reported in the original paper for measurements of helically loaded "Helix-II" resonators at various harmonics.

b Data extracted from the published plots for " $120^{\circ} \mathrm{C}$ baked" cavities. Different frequencies correspond to different elliptical cavities.

"The data correspond to the $A / B$ families identified in the original paper ("oxidized Cu"/"oxide-free Cu").

which results in

$\operatorname{Re}\left[A_{1}\right]=\frac{\omega_{o} x_{o}}{\omega^{2}+\omega_{o}^{2}} \frac{1}{T} \int_{0}^{T}\left(\omega_{o} \cos \omega t-\omega \sin \omega t\right)\left|\frac{x_{o}(t)}{x_{o}}\right| \frac{x_{o}(t)}{x_{o}} d t$.

The calculation of the integrals in Eqs. (14) and (18) is drafted in the Appendix. Combining the result of Eq. (A6) with the expressions of Eq. (5), we finally obtain

$$
R_{f l}\left(B_{r f o}\right)=R_{f l}(0)\left[1+\frac{16 \alpha}{3 \pi} \frac{\omega_{0}^{2}}{\left(\omega^{2}+\omega_{o}^{2}\right)}\right]
$$

with

$$
R_{f l}(0)=R_{n} \frac{B_{o}}{B_{c 2}} \frac{\omega^{2}}{\omega^{2}+\omega_{o}^{2}} .
$$

The parameter $\alpha$ contains the dependence on $B_{r f 0}$ :

$\alpha=\frac{\gamma x_{o}}{k}=\frac{\gamma \phi_{o} J_{r f o}}{k \eta \sqrt{\omega^{2}+\omega_{o}^{2}}}=\frac{\gamma}{k} \frac{R_{n}}{\mu_{o}} \frac{B_{r f o}}{B_{c 2}} \frac{1}{\sqrt{\omega^{2}+\omega_{o}^{2}}}$.

For $\alpha=0$, i.e., for $\gamma=0$ or $B_{r f o}=0$, the GR result of Eq. (6) is reproduced.

Our nonlinear model also reproduces the phenomenological Eq. (1) with

$$
\begin{gathered}
R_{f l}^{0}=\frac{R_{n}}{B_{c 2}} \frac{\omega^{2}}{\omega^{2}+\omega_{o}^{2}}, \\
R_{f l}^{1}=R_{f l}^{o} \frac{16}{3 \pi} \frac{\gamma}{k} \frac{R_{n}}{\mu_{o} B_{c 2}} \frac{1}{\sqrt{\omega^{2}+\omega_{o}^{2}}} \frac{\omega_{o}^{2}}{\left(\omega^{2}+\omega_{o}^{2}\right)} .
\end{gathered}
$$

\section{COMPARISON WITH EXPERIMENTAL RESULTS}

The model presented in the previous section predicts a linear dependence of the flux sensitivity $S=R_{f l} / B_{0}$ on the amplitude of the rf field $B_{r f 0}$. This is indeed observed in several experiments, on both bulk $\mathrm{Nb}$ cavities $[3,5,7]$ and $\mathrm{Nb} / \mathrm{Cu}$ thin film cavities [6,26,27], and allows expressing the flux sensitivity as in Eq. (1). The coefficients of flux sensitivity and rf field dependence measured in these experiments are reported in Table I.

In practice, however, this is a somewhat trivial result, since the shape of the nonquadratic pinning potential (nonlinear pinning force) has been selected to reproduce this result. A higher order of the potential would have naturally resulted in a higher-order rf field dependence.

From the experimental data, it is always possible to deduce the value of the nonlinear parameter $\alpha$ and verify whether this term is small enough to be compatible with the adopted perturbative approach. From Eq. (19) and in the hypothesis $\omega<\omega_{o}$, certainly adequate for $\mathrm{Nb}$ films, the maximum rf field $B_{r f 0}^{\max }$ for which the minimum condition $\alpha<0.5$ is satisfied corresponds to $R_{f l}\left(B_{r f 0}^{\max }\right) \lesssim 2 R_{f l}(0)$. As an example, for the $\mathrm{Nb} / \mathrm{Cu}$ films reported in Fig. 2, this is true up to a $B_{r f 0} \leq 5 \mathrm{mT}$; however, the range of the linear dependence of the flux sensitivity on $B_{r f 0}$ extends much beyond this value. We should nevertheless underline that at the largest amplitudes the flux sensitivity deviates from linearity.

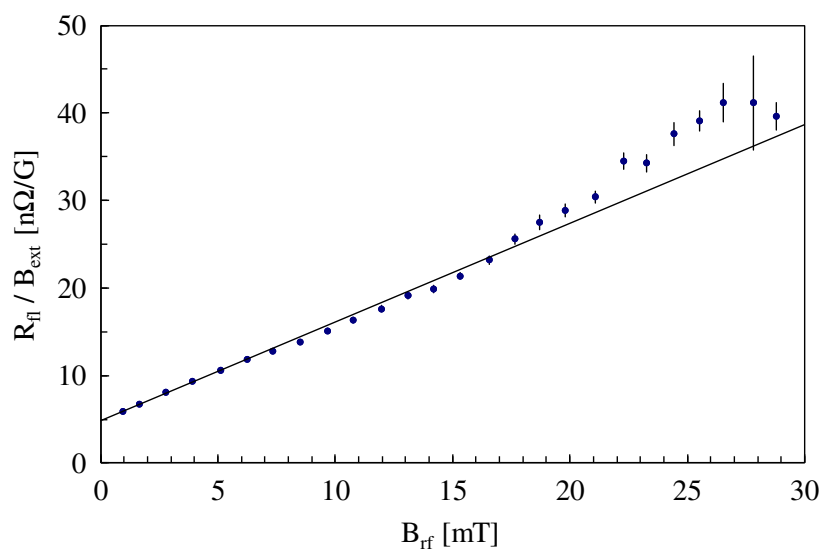

FIG. 2. The dependence on $B_{r f}$ of $R_{f l}(1.7 K) / B_{\text {ext }}$ for $\mathrm{Nb} / \mathrm{Cu}$ cavities. The line is a fit to the data of the form $R_{f l}^{0}+R_{f l}^{1} B_{r f}$. (Reprinted from Ref. [6] with STM permission.) 


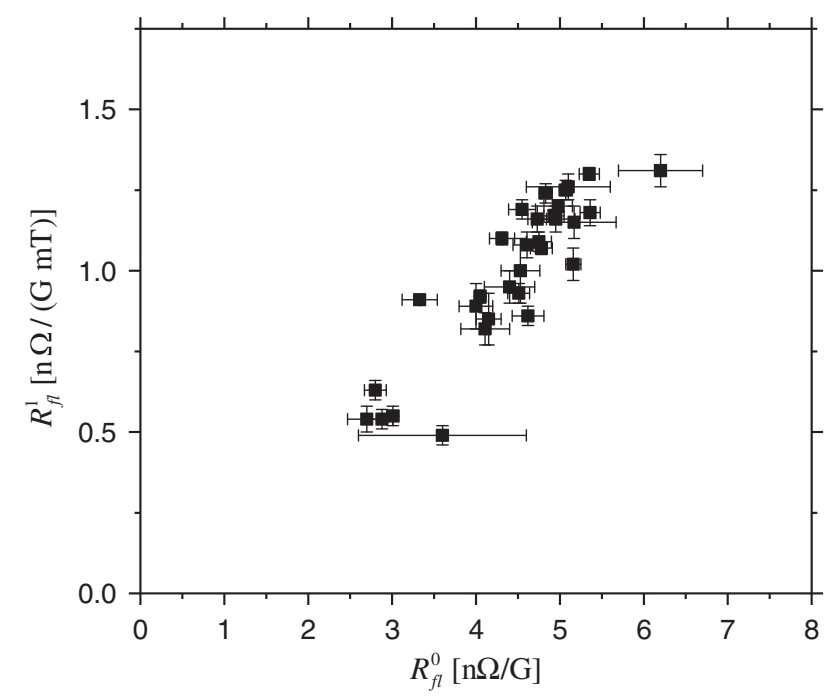

FIG. 3. Correlation plot of $R_{f l}^{o}$ and $R_{f l}^{1}$ for $\mathrm{Nb} / \mathrm{Cu}$ films at $1500 \mathrm{MHz}[6]$.

Our nonlinear model predicts also other nontrivial experimental results. Indeed, Eq. (23) shows a proportionality between $R_{f l}^{o}$ and $R_{f l}^{1}$ as observed on bulk $\mathrm{Nb}[3,6]$, on $\mathrm{Nb} / \mathrm{Cu}$ thin films $[6,27]$, and also on $\mathrm{Nb}_{3} \mathrm{Sn}$ films grown by thermal diffusion of $\mathrm{Sn}$ vapors inside $\mathrm{Nb}$ cavities [28]. Data extracted from Ref. [6] for several $\mathrm{Nb} / \mathrm{Cu}$ cavities coated with different sputter gases $(\mathrm{Ar}, \mathrm{Kr}$, and $\mathrm{Xe}$ ) produced at CERN are reported in Fig. 3, which illustrates well the predicted behavior.

The present model can also describe the frequency dependence of the effect of trapped flux. From Eqs. (22) and (23), we can write

$$
R_{f l}^{1} \propto \frac{1}{\sqrt{\omega^{2}+\omega_{o}^{2}}}\left(\frac{\omega_{o} \omega}{\omega^{2}+\omega_{o}^{2}}\right)^{2} .
$$

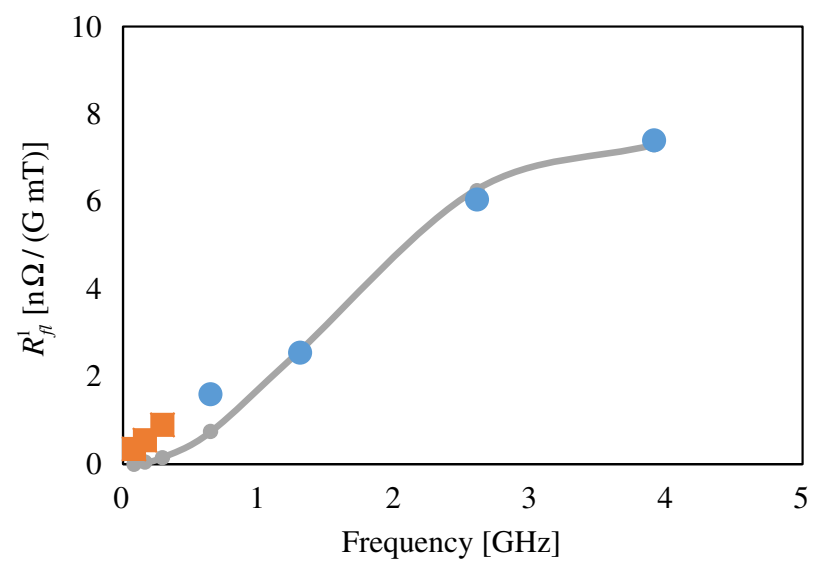

FIG. 4. Dependence of $R_{f l}^{1}$ on the frequency. Data on Helix-II low-frequency resonators are from Ref. [3] (squares) and on $120^{\circ} \mathrm{C}$ baked elliptical cavities from Ref. [7] (circles). The gray line is a best fit to the data, resulting in a depinning frequency $\nu_{0}=\omega_{0} / 2 \pi=4.6 \mathrm{GHz}$.
This dependence is plotted in Fig. 4 for $\omega \leq \omega_{o}$, fitted to the available data from recent experiments on " $120^{\circ} \mathrm{C}$ baked" bulk $\mathrm{Nb}$ cavities [7] and to older experimental results at a lower frequency which date back to the very early developments of SRF using low-RRR $\mathrm{Nb}$ [3], also reported in the figure.

One should be aware, however, that Eq. (24) predicts a decrease of $R_{f l}^{1}$ vs $\omega$ for $\omega \geq \omega_{o}$ not observed in the experiments.

\section{CONCLUSIONS}

We have shown that, by inserting a simple nonharmonic pinning potential in the GR equations, we can correctly reproduce the linear dependence on $B_{r f o}$ of the losses induced by an external trapped magnetic field in SRF cavities. A proportionality between $R_{f l}^{o}$ and $R_{f l}^{1}$ follows naturally from this approach. In the limit $\omega<\omega_{0}$, the frequency dependence of $R_{f l}^{1}$ observed in the experiments can also be reproduced.

We are aware that our simple model cannot reproduce all the large body of experimental evidence; however, its good capability of describing some of the main features of the flux sensitivity could be the basis for a more complete theoretical treatment based on more refined models, which would include the specific mechanisms producing the nonlinear pinning force.

\section{ACKNOWLEDGMENTS}

The authors acknowledge Alex Gurevich (Old Dominion University) for his helpful suggestions on the techniques for solving nonlinear equations and the valuable comments on the manuscript. The authors also acknowledge several stimulating discussions with Akira Miyazaki and Walter Venturini Delsolaro (CERN). R. V. has been supported by the INFN V Group Experiment ISIDE.

\section{APPENDIX}

From Eqs. (14) and (18), we have

$$
\begin{aligned}
P_{s f l}= & P_{s f l 0}+P_{s f l 1} \\
= & d \phi_{o} J_{r f o} \omega x_{o} \cdot\left\{\frac{1}{T} \int_{0}^{T} \cos (\omega t-\varphi) \sin \omega t d t\right. \\
& +\frac{\alpha \omega_{o}}{\left(\omega^{2}+\omega_{o}^{2}\right)}\left[\omega \frac{1}{T} \int_{0}^{T} \sin \omega t|\sin (\omega t-\varphi)| \sin (\omega t-\varphi) d t\right. \\
& \left.\left.-\omega_{o} \int_{0}^{T} \cos \omega t|\sin (\omega t-\varphi)| \sin (\omega t-\varphi) d t\right]\right\} . \quad(\mathrm{A} 1)
\end{aligned}
$$

The integrals in Eq. (A1) can easily be computed, giving, respectively, 


$$
\frac{1}{T} \int_{0}^{T} \cos (\omega t-\varphi) \sin \omega t d t=\frac{1}{2} \sin \varphi
$$

$\frac{1}{T} \int_{0}^{T} \sin \omega t|\sin (\omega t-\varphi)| \sin (\omega t-\varphi) d t=\frac{4}{3 \pi} \cos \varphi$,

$\frac{1}{T} \int_{0}^{T} \cos \omega t|\sin (\omega t-\varphi)| \sin (\omega t-\varphi) d t=-\frac{4}{3 \pi} \sin \varphi$.

Then, using the result of Eq. (4) for $x_{o}$ and $\varphi$, we get

$$
P_{s f l}=\frac{1}{2} \frac{d \phi_{o}^{2} J_{r f o}^{2}}{\eta} \frac{\omega^{2}}{\omega^{2}+\omega_{o}^{2}}\left[1+\frac{16}{3 \pi} \alpha \frac{\omega_{o}^{2}}{\left(\omega^{2}+\omega_{o}^{2}\right)}\right] .
$$

If we have $n$ vortices per unit surface characterized by the same depinning current, since the overall power dissipated per unit surface is obviously $P_{s}=n P_{s f l}$ and remembering that $B_{0}=n \phi_{0}, H_{r f o}=d J_{r f o}, \eta=\phi_{o} B_{c 2} / \rho_{n}$, and $R_{n}=\rho_{n} / d$ (for a thin slab), we finally obtain

$P_{s}=\frac{1}{2} R_{n} \frac{B_{o}}{B_{c 2}} \frac{\omega^{2}}{\omega^{2}+\omega_{o}^{2}}\left[1+\frac{16 \alpha}{3 \pi} \frac{\omega_{0}^{2}}{\left(\omega^{2}+\omega_{o}^{2}\right)}\right] H_{r f o}^{2}$.

[1] S. Aull, O. Brunner, A. Butterworth, and N. Schwerg, Material options for the superconducting rf system of the Future Circular Collider, Report No. CERN-ACC-20180019, https://cds.cern.ch/record/2625126.

[2] LCLS-II Design Study Group, LINAC Coherent Light Source II (LCLS-II) Project Final Design Report No. LCLSII-1.1-DR-0251-R0, SLAC National Accelerator Laboratory, 2015, https://www.osti.gov/biblio/1442520.

[3] B. Piosczyk, P. Kneisel, O. Stoltz, and J. Halbritter, Investigation of additional losses in superconducting niobium cavities due to frozen-in flux, IEEE Trans. Nucl. Sci. 20, 108 (1973).

[4] B. Cauvin, M. Coret, J. P. Fouan, J. Girard, J. L. Girma, Ph. Leconte, Y. Lussignol, R. Moreau, J. P. Passérieux, G. Ramstein, and L. Wartski, Fabrication, tests, and RF control of the 50 superconducting resonators of the Saclay heavy ion Linac, in Proceedings of the Third Workshop on rf Superconductivity (Argonne National Laboratories, Argonne, 1987), pp. 379-388, http://accelconf.web.cern .ch/AccelConf/srf87/papers/srf87d02.pdf.

[5] G. Arnolds-Mayer and W. Weingarten, Comparative measurements on niobium sheet and sputter coated cavities, IEEE Trans. Magn. 23, 1620 (1987).

[6] C. Benvenuti, S. Calatroni, I. E. Campisi, P. Darriulat, M. A. Peck, R. Russo, and A-M. Valente, Study of the surface resistance of superconducting niobium films at 1.5 GHz, Physica (Amsterdam) 316C, 153 (1999).
[7] M. Checchin, M. Martinello, A. Grassellino, S. Aderhold, S. K. Chandrasekaran, O.S. Melnychuk, S. Posen, A. Romanenko, and D. A. Sergatskov, Frequency dependence of trapped flux sensitivity in SRF cavities, Appl. Phys. Lett. 112, 072601 (2018).

[8] H. Padamsee, RF Superconductivity: Science, Technology and Applications (Wiley-VCH, Weinheim, 2009).

[9] J.-M. Vogt, O. Kugeler, and J. Knobloch, Impact of cooldown conditions at Tc on the superconducting rf cavity quality factor, Phys. Rev. ST Accel. Beams 16, 102002 (2013).

[10] A. Romanenko, A. Grassellino, O. Melnychuk, and D. A. Sergatskov, Dependence of the residual surface resistance of superconducting radio frequency cavities on the cooling dynamics around Tc, J. Appl. Phys. 115, 184903 (2014).

[11] A. Romanenko, A. Grassellino, A. C. Crawford, D. A. Sergatskov, and O. Melnychuk, Ultra-high quality factors in superconducting niobium cavities in ambient magnetic fields up to 190 mG, Appl. Phys. Lett. 105, 234103 (2014).

[12] M. Martinello, A. Grassellino, M. Checchin, A. Romanenko, O. Melnychuk, D. A. Sergatskov, S. Posen, and J.F. Zasadzinski, Effect of interstitial impurities on the field dependent microwave surface resistance of niobium, Appl. Phys. Lett. 109, 062601 (2016).

[13] D. Gonnella, J. Kaufman, and M. Liepe, Impact of nitrogen doping of niobium superconducting cavities on the sensitivity of surface resistance to trapped magnetic flux, J. Appl. Phys. 119, 073904 (2016).

[14] J. I. Gittleman and B. Rosenblum, Radio-Frequency Resistance in the Mixed State for Subcritical Currents, Phys. Rev. Lett. 16, 734 (1966).

[15] R. Marcon, R. Fastampa, M. Giura, and E. Silva, Vortexmotion dissipation in high-Tc superconductors at microwave frequencies, Phys. Rev. B 43, 2940 (1991).

[16] É. A. Pashitskiǔ and V. I. Vakaryuk, Pinning of Abrikosov vortices on dislocations and the critical current in high-temperature superconductors, Low Temp. Phys. 28, 11 (2002).

[17] H. Padamsee, J. Knobloch, and T. Hays, RF Superconductivity for Accelerators (Wiley, New York, 1998), ISBN: 9780471154327.

[18] D. Janjuševic, M. S. Grbic, M. Pozek, A. Dulcic, D. Paar, B. Nebendahl, and T. Wagner, Microwave response of thin niobium films under perpendicular static magnetic fields, Phys. Rev. B 74, 104501 (2006).

[19] G. Blatter, M. V. Feigel'man, V. B. Geshkenbein, A. I. Larkin, and V. M. Vinokur, Vortices in high-temperature superconductors, Rev. Mod. Phys. 66, 1125 (1994).

[20] S. Calatroni and R. Vaglio, Surface resistance of superconductors in the presence of a dc magnetic field: Frequency and field intensity limits, IEEE Trans. Appl. Supercond. 27, 3500506 (2017).

[21] M. Checchin, M. Martinello, A. Grassellino, A. Romanenko, and J. F. Zasadzinski, Electron mean free path dependence of the vortex surface impedance, Supercond. Sci. Technol. 30, 034003 (2017).

[22] A. Gurevich and G. Ciovati, Dynamics of vortex penetration, jumpwise instabilities, and nonlinear surface resistance of type-II superconductors in strong rf fields, Phys. Rev. B 77, 104501 (2008). 
[23] A. Gurevich and G. Ciovati, Effect of vortex hotspots on the radio-frequency surface resistance of superconductors, Phys. Rev. B 87, 054502 (2013).

[24] D. B. Liarte, D. Hall, P. N. Koufalis, A. Miyazaki, A. Senanian, M. Liepe, and J. P. Sethna, Vortex dynamics and losses due to pinning: Dissipation from trapped magnetic flux in resonant superconducting radio-frequency cavities, arXiv:1808.01293.

[25] L. Embon et al., Probing dynamics and pinning of single vortices in superconductors at nanometer scales, Sci. Rep. 5, 7598 (2015).
[26] A. Miyazaki and W. Venturini Delsolaro, Two different origins of the Q-slope problem in superconducting niobium film cavities for a heavy ion accelerator at CERN, arXiv: 1812.04658.

[27] C. Benvenuti, S. Calatroni, P. Darriulat, M. A. Peck, and A.-M. Valente, Fluxon pinning in niobium films, Physica (Amsterdam) 351C, 429 (2001).

[28] D. Hall, New insights into the limitations on the efficiency and achievable gradients in Nb3Sn SRF cavities, Ph.D. thesis, Cornell University, 2017, https://www.classe.cornell.edu/ rsrc/Home/Research/SRF/SrfDissertations/Daniel_Hall_ PhD_Thesis_Final.pdf. 